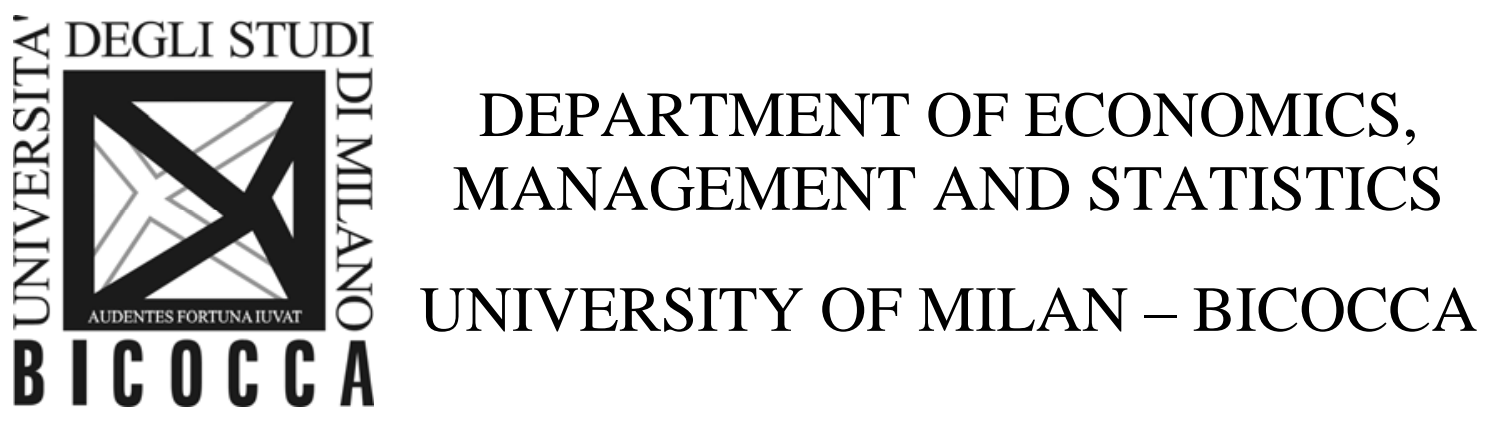

DEMS WORKING PAPER SERIES

\title{
Dynamics in a nonlinear Keynesian good market model
}

Ahmad Naimzada, Marina Pireddu

No. 254 - September 2013

Dipartimento di Economia, Metodi Quantitativi e Strategie di Impresa Università degli Studi di Milano - Bicocca http://dems.unimib.it/ 


\title{
Dynamics in a nonlinear Keynesian good market model
}

\author{
Ahmad Naimzada ${ }^{\text {a*}}$, Marina Pireddu ${ }^{\mathrm{b} \dagger}$ \\ ${ }^{a}$ Dept. of Economics, Quantitative Methods and Management, University of Milano-Bicocca, Milan \\ ${ }^{\mathrm{b}}$ Dept. of Mathematics and Applications, University of Milano-Bicocca, Milan
}

\begin{abstract}
In this paper we show how a rich variety of dynamical behaviors can emerge in the standard Keynesian income-expenditure model when a nonlinearity is introduced, both in the cases with and without endogenous government spending. A specific sigmoidal functional form is used for the adjustment mechanism of income with respect to the excess demand, in order to bound the income variation. With the aid of analytical and numerical tools, we investigate the stability conditions, bifurcations, as well as periodic and chaotic dynamics. Globally, we study multistability phenomena, i.e., the coexistence of different kinds of attractors.
\end{abstract}

Keywords: nonlinearities; complex dynamics; Keynesian model; business cycle; fiscal policy.

AMS classification: 37N40, 39A28, 39A60, 91B55

JEL classification: C62, E12, E32, E62

*Mailing address: University of Milano-Bicocca, U7 Building, Via Bicocca degli Arcimboldi 8, 20126 Milano, Italy. Tel.: +390264485813; fax: +390264483085. E-mail address: ahmad.naimzada@unimib.it

${ }^{\dagger}$ Corresponding author at: University of Milano-Bicocca, U5 Building, Via Cozzi 53, 20125 Milano, Italy. Tel.: +39 0264485729; fax: +390264485705. E-mail address: marina.pireddu@unimib.it 


\section{Introduction}

Instabilities are known, both empirically and theoretically, to be features of all markets: the product markets, the labor market, and the financial markets. Persistent irregularities exhibited by macroeconomic variables are usually attributed to random shocks (see for instance $[8,9]$ ). An alternative explanation is based on phenomena caused by nonlinearities in the deterministic frameworks (see e.g. $[5,7,11,12,13]$ ). For instance, in the Keynesian IS-LM model, when introducing nonlinearities and lags, complex dynamics have been obtained in $[1,2,3]$.

Our aim consists in showing that even simpler Keynesian frameworks such as the income-expenditure setting are able to generate interesting complex dynamics. In fact the deterministic standard textbook Keynesian income-expenditure model is not able to produce rich dynamical behaviors given the linearity of the economic relations and the linearity of the income adjustment mechanism. On the other hand in this paper we show how the introduction of a simple nonlinearity may produce interesting dynamics. More precisely, we consider a specific sigmoidal functional form for the adjustment mechanism of income with respect to the excess demand, in order to bound the income variation. In particular, in our model we introduce parameters representing the reactivity and the bounds of the income adjustment mechanism. Such a setting is then extended by endogenizing the government expenditure via the introduction of parameters representing the reactivity and the income target of the fiscal policy authority. With the aid of analytical and numerical tools we show for the model without endogenous public expenditure that increasing the reactivity in the adjustment mechanism destabilizes the system, while decreasing the bounds in the income adjustment mechanism has a stabilizing effect. Such stabilization occurs via a sequence of period-halving bifurcations. For the extended model with public expenditure we find that increasing the reactivity with respect to the difference between the income target and the current target has a destabilizing effect and that the steady state value increases if the reactivity increases, if and only if the income target is larger than the income steady state without public expenditure. From an economic point of view, the latter result means that, independently of the nature of the attractor, the weight of the endogenous public expenditure may increase the value of income, if the target of the fiscal policy authority is sufficiently large. We also find that the income target has no influence on the stability properties of the system, while it has a direct influence on the steady state position. Globally, we find that endogenizing the public expenditure may imply multistability, i.e., the coexistence of different kinds of attractors. For both cases, with and without public expenditure, we show that, differently from the linear case in which local instability implies diverging trajectories, with the introduction of the sigmoidal adjustment mechanism in the instability regime we have the emergence of an absorbing interval, i.e., an invariant interval which eventually captures all forward trajectories. Moreover, we show the existence of chaotic dynamics in the sense of Li and Yorke [10] for both the scenarios 
considered.

The remainder of the paper is organized as follows. In Section 2 we introduce the model with exogenous government spending. In Section 3 we present analytical and numerical local results for this setting, and we show the existence of an absorbing interval. In Section 4 we investigate analytically the first flip bifurcation and the existence of Li-Yorke chaos. In Section 5 we introduce the model with endogenous government spending and show how the results in Sections 3 and 4 can be adapted to that new framework. In Section 6 we present a global scenario with multistability phenomena. Finally, in Section 7 we draw some conclusions and discuss our results.

\section{The model}

We consider a model with a Keynesian good market of a closed economy with public intervention. It is assumed that investment and government expenditures are exogenously given and that consumption has an autonomous component, as well as a term depending on income. The basic static model is as follows:

$$
Y=C+I+G
$$

with

$$
I=\bar{I}, \quad G=\bar{G}, \quad C=\bar{C}+c Y,
$$

where $Y$ is aggregate income, $C$ is aggregate consumption, $I$ is aggregate investment and $G$ is government expenditure. Investment and government expenditures are exogenous and equal to $\bar{I}$ and $\bar{G}$, respectively. In the consumption function, $\bar{C}$ is autonomous consumption and $c \in(0,1)$ is the marginal propensity to consume. Equation (2.1) is the Keynesian equilibrium condition.

In a dynamic framework we assume a dependence of consumption at time $t$ on the income in the same time period, i.e., $C_{t}=\bar{C}+c Y_{t}$. The dynamic behavior in the real economy is represented by an adjustment mechanism depending on the excess demand. If aggregate excess demand is positive (negative), production increases (decreases), that is, income $Y_{t+1}$ in period $t+1$ is defined in the following way

$$
Y_{t+1}=Y_{t}+\gamma g\left(E_{t}\right)
$$

where $\gamma>0$ is the real market speed of adjustment between demand and supply, $g$ is an increasing function with $g(0)=0$ and $E_{t}=Z_{t}-Y_{t}$ is the excess demand, with $Z_{t}$ the aggregate demand in a closed economy, defined as

$$
Z_{t}=C_{t}+I_{t}+G_{t}
$$

We stress that, due to the properties of the map $g$, the dynamical system generated by (2.2) has a unique steady state, corresponding to $E_{t}=0$, whose expression is given 
by

$$
Y^{*}=\frac{A}{1-c},
$$

where $A=\bar{C}+\bar{I}+\bar{G}$ is aggregate autonomous expenditure and $\frac{1}{1-c}$ is the Keynesian multiplier (see [4]).

Imposing a linear adjustment mechanism, the corresponding dynamical system is described by equation (2.2) with $g$ equal to the identity map, which can be rewritten as follows:

$$
\Delta_{t+1}:=Y_{t+1}-Y_{t}=\gamma E_{t},
$$

where $\Delta_{t+1}$ represents the income variation. We represent such framework in Figure 1.

Making all terms explicit, in the linear case equation (2.2) can be also rewritten as follows:

$$
Y_{t+1}=Y_{t}+\gamma\left(Z_{t}-Y_{t}\right)=Y_{t}+\gamma\left(\bar{C}+\bar{I}+\bar{G}-(1-c) Y_{t}\right)=Y_{t}+\gamma\left(A-(1-c) Y_{t}\right) .
$$

In order to have a converging behavior towards $Y^{*}$, we need

$$
-1<1-\gamma(1-c)<1
$$

which is satisfied for $\gamma<\frac{2}{1-c}$. For a larger value of $\gamma$ we have instead a diverging behavior, while for $\gamma=\frac{2}{1-c}$ we have infinitely many period-two cycles.

Looking again at Figure 1 , we observe that $\Delta_{t+1}$ may grow unboundedly and, in particular, when $E_{t}$ limits to $\pm \infty$, the same does $\Delta_{t+1}$. However, this is an unrealistic assumption because of the material constraints in the production side of an economy.

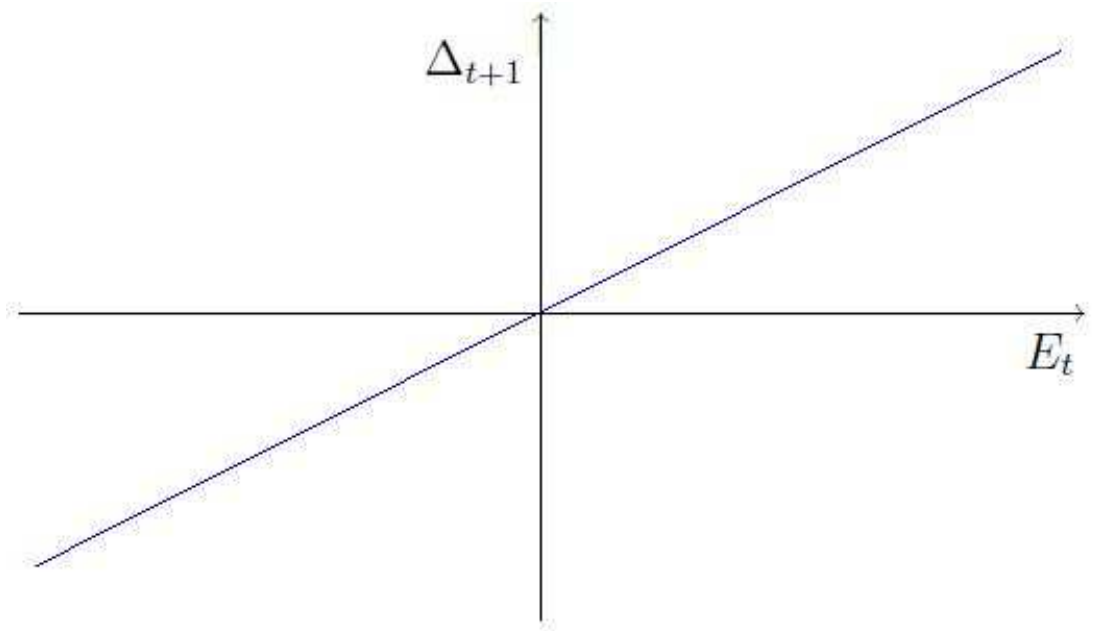

Figure 1: The graph of $\Delta_{t+1}$ as a function of $E_{t}$ in the linear case in (2.5).

Assuming instead that the adjustment mechanism is $S$-shaped, we specify the function $g$ as 


$$
g\left(E_{t}\right)=a_{2}\left(\frac{a_{1}+a_{2}}{a_{1} e^{-E_{t}}+a_{2}}-1\right)
$$

with $a_{1}, a_{2}$ positive parameters.

With such choice, $g$ is increasing and $g(0)=0$. Moreover, it is bounded from below by $-a_{2}$ and from above by $a_{1}$. Thus the income variations are gradual and this prevents the real market from diverging and it may create a real oscillator.

Inserting $Z_{t}$ from (2.3) into (2.2) and recalling the definition of $\Delta_{t+1}$ in (2.5), we obtain the dynamic equation of the real market in the nonlinear framework

$$
\Delta_{t+1}=\gamma a_{2}\left(\frac{a_{1}+a_{2}}{a_{1} e^{-E_{t}}+a_{2}}-1\right)=\gamma a_{2}\left(\frac{a_{1}+a_{2}}{a_{1} e^{-\left(A-Y_{t}(1-c)\right)}+a_{2}}-1\right)
$$

that we depict in Figure 2 and that generates the dynamical system we are going to analyze in the Sections 3 and 4.

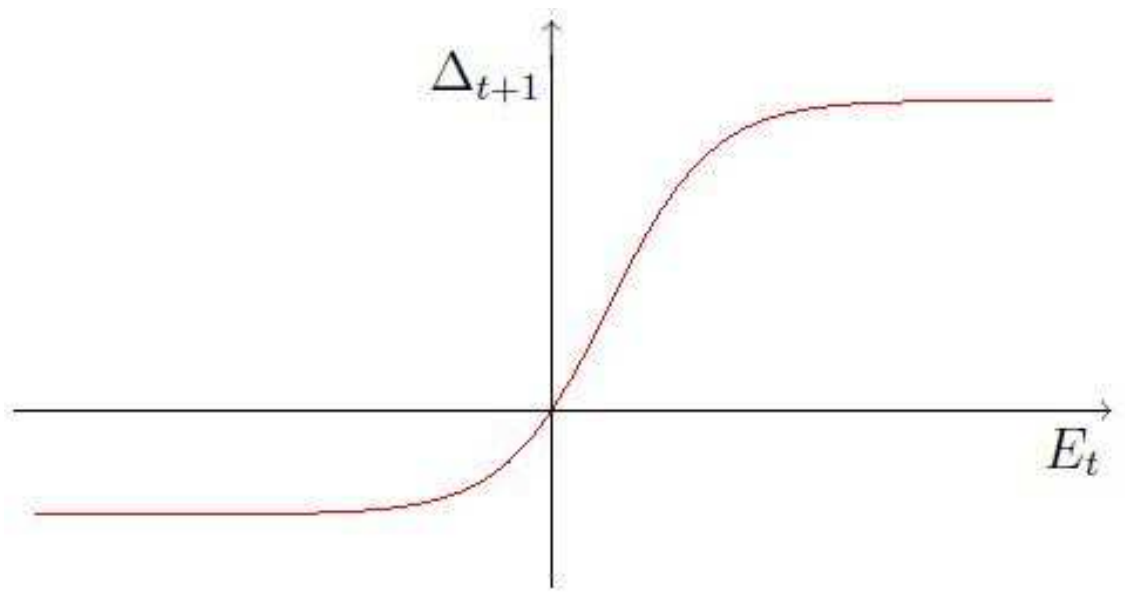

Figure 2: The graph of $\Delta_{t+1}$ as a function of $E_{t}$ in the nonlinear case in (2.7).

\section{Local analysis and the absorbing interval}

In this section we analyze the stability of the steady state in the nonlinear case. Moreover, we show the presence of an absorbing interval eventually attracting all forward trajectories.

In view of the subsequent analysis, it is expedient to introduce the map $F: \mathbb{R}_{+} \rightarrow \mathbb{R}$ defined as

$$
F(Y)=Y+\gamma a_{2}\left(\frac{a_{1}+a_{2}}{a_{1} e^{-(A-Y(1-c))}+a_{2}}-1\right),
$$

associated to the dynamic equation in (2.7). 
Proposition 3.1 The steady state $Y^{*}$ in (2.4) is stable if and only if

$$
\gamma(1-c)<\frac{2\left(a_{1}+a_{2}\right)}{a_{1} a_{2}} .
$$

Proof. The result immediately follows by solving the chain of inequalities $-1<$ $F^{\prime}\left(Y^{*}\right)<1$ with respect to $\gamma(1-c)$. In fact, it is immediate to find that $F^{\prime}\left(Y^{*}\right)=$ $1-\frac{\gamma a_{1} a_{2}(1-c)}{a_{1}+a_{2}}$ and thus, in order to get the stability of the steady state, we only need to impose $F^{\prime}\left(Y^{*}\right)>-1$.

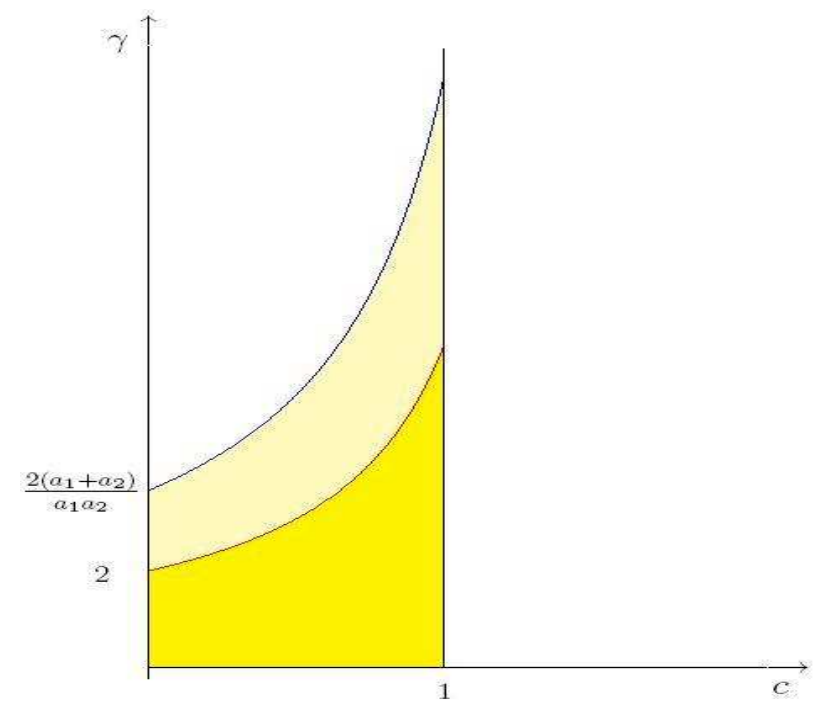

Figure 3: The stability regions in the $(c, \gamma)$-plane for the linear and nonlinear cases (in dark yellow and pale yellow, respectively) with $a_{1}=1$ and $a_{2}=1.2$.

At first, we stress that, recognizing in $s=1-c$ the propensity to save, it is possible to rewrite the stability condition in Proposition 3.1 as $\gamma s<\frac{2\left(a_{1}+a_{2}\right)}{a_{1} a_{2}}$. Hence it is evident that an increase in $\gamma$ or $s$ has a destabilizing effect.

Considering instead the stability condition in terms of $\gamma$ only, we get $\gamma<\frac{2\left(a_{1}+a_{2}\right)}{a_{1} a_{2}(1-c)}$. Comparing then the thresholds for $\gamma$ in the linear and nonlinear cases, i.e., $\bar{\gamma}=\frac{2}{(1-c)}$ and $\overline{\bar{\gamma}}=\frac{2\left(a_{1}+a_{2}\right)}{a_{1} a_{2}(1-c)}$, respectively, we find that $\bar{\gamma}<\overline{\bar{\gamma}}$ if and only if $a_{1}+a_{2}>a_{1} a_{2}$. In other terms, since $\frac{\left(a_{1}+a_{2}\right)}{a_{1} a_{2}}=\frac{1}{a_{1}}+\frac{1}{a_{2}}$, we have that $\bar{\gamma}<\overline{\bar{\gamma}}$ when $a_{1}$ and $a_{2}$ are small enough. This is the case in Figure 3 , where we represent the stability regions in the $(c, \gamma)$-plane for the linear and nonlinear formulations. An intuition of the fact that small values for $a_{1}$ and $a_{2}$ allow to enlarge the stability region can be obtained by observing that, when reducing $a_{1}$ and $a_{2}$, we decrease the current variation of output. In fact, $a_{1}$ and $a_{2}$ appear in the expression of the asymptotes, whose equations in the $(x, y)$-plane are $y=x-\gamma a_{2}$ for $x \rightarrow+\infty$ and $y=x+\gamma a_{1}$ for $x \rightarrow-\infty$, and, as it is immediate to verify, such straight lines get closer when $a_{1}$ and $a_{2}$ decrease. 


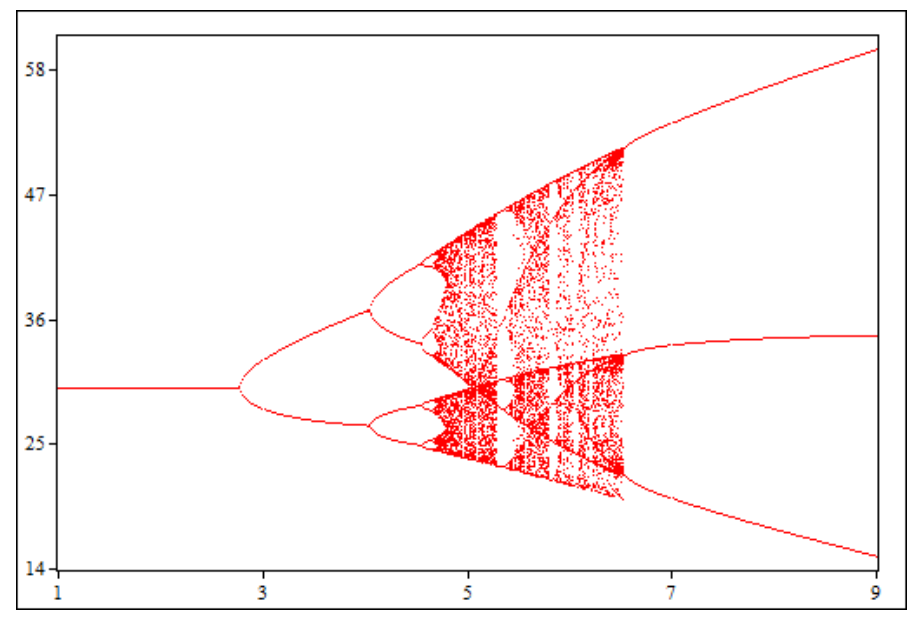

Figure 4: The bifurcation diagram w.r.t. $\gamma \in[1,9]$ for the map $F$ with $A=12$, $a_{1}=5, a_{2}=2.8$ and $c=0.6$.

Looking again at the shape of the stability region in Figure 3 for the linear (nonlinear) case, we observe that when $\gamma<2\left(\gamma<\frac{2\left(a_{1}+a_{2}\right)}{a_{1} a_{2}}\right)$ the steady state is stable for any $c$ and that, when $\gamma$ increases, we need values for $c$ closer and closer to 1 in order to stabilize $Y^{*}$. Hence increasing $\gamma$ has a destabilizing effect, both in the linear and nonlinear cases. In Figure 4 we depict the bifurcation diagram with respect to $\gamma$ in the nonlinear framework, which confirms the destabilizing role of $\gamma$. In particular we stress that, after the chaotic regime following the destabilization of the fixed point, it is possible to have again a periodic cycle, like in Figure 4, where we end up with a period-three orbit.

In Figure 5 we depict the bifurcation diagram with respect to $\gamma$ in the nonlinear framework, for the same parameter values in Figure 3, except for a bit larger value of $a_{2}$. This change makes the loss of stability of the steady state happen for values of $\gamma$ smaller with respect to Figure 3, confirming what said above about the destabilizing role of increasing $a_{2}$ (and $a_{1}$ ).

Since, as observed above, the steady states for the linear and nonlinear scenarios coincide with $Y^{*}$, we depict in Figure 6 the graph of the maps corresponding to those two cases in a neighborhood of $Y^{*}$, i.e.,

$$
f: \mathbb{R}_{+} \rightarrow \mathbb{R}, \quad f(Y)=Y+\gamma(A-(1-c) Y)
$$

and $F$ in (3.1), respectively, when $a_{1}+a_{2}=a_{1} a_{2}$, that is, when also the stability thresholds for the linear and nonlinear scenarios coincide.

Starting from this framework in which $a_{1}+a_{2}=a_{1} a_{2}$, we depict in Figure 7 the bifurcation diagram for the map $F$ with respect to $a_{1}=a_{2} \in[0,2]$, which shows that decreasing $a_{1}=a_{2}$ from 2 (value for which the condition $a_{1}+a_{2}=a_{1} a_{2}$ is satisfied and both systems are unstable) to 0 has a stabilizing effect. 


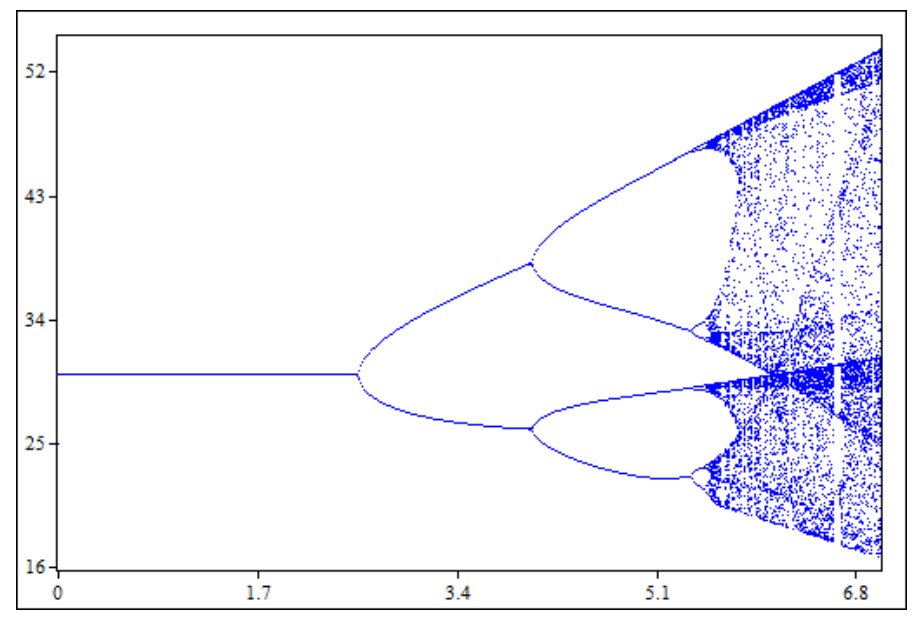

Figure 5: The bifurcation diagram w.r.t. $\gamma \in[0,7]$ for the map $F$ with $A=12$, $a_{1}=5, a_{2}=3.2$ and $c=0.6$.

Similarly, in Figure 8 we present the bifurcation diagram for the map $F$ with respect to $a_{1} \in[0,4]$, when $a_{2}=\frac{4}{3}$. That picture shows that, if we start with $a_{1}=4$ and $a_{2}=\frac{4}{3}$ (so that $a_{1}+a_{2}=a_{1} a_{2}$ ) and we then decrease $a_{1}$ from 4 to 0 , we obtain again a stabilizing effect.

We now describe some further dynamical features for the map $F$ in the next result. More precisely, we show the existence of an absorbing interval and thus, differently from the linear case, in which local instability implies diverging trajectories (see [4]), in our framework local instability may imply periodic and chaotic orbits.

Proposition 3.2 If the map $F$ in (3.1) is increasing, then the generated dynamical system is globally stable. Else ${ }^{1}$, call $m$ and $M$ the unique positive local minimum point and local maximum point of the map $F$, respectively, and set $m^{\prime}:=F(m)$ and $M^{\prime}:=F(M)$. Then the compact interval $I=\left[\mathrm{m}^{\prime}, M^{\prime}\right]$ is "globally absorbing", i.e., for all $\bar{x} \in \mathbb{R}_{+}$there exists $\bar{n} \in \mathbb{N}$ such that $F^{\bar{n}}(\bar{x}) \in I$ and for any $x \in I, F^{n}(x) \in I$, for all $n \in \mathbb{N}$.

${ }^{1}$ It is possible to show that no other scenarios may arise for the map $F$. In fact

$$
F(0)=\gamma a_{2}\left(\frac{a_{1}+a_{2}}{a_{1} e^{-A}+a_{2}}-1\right)>\gamma a_{2}\left(\frac{a_{1}+a_{2}}{a_{1}+a_{2}}-1\right)=0
$$

and

$$
F^{\prime}(0)=1-\frac{\gamma a_{1} a_{2}\left(a_{1}+a_{2}\right) e^{-A}(1-c)}{\left(a_{1} e^{-A}+a_{2}\right)^{2}},
$$

which is positive for any $A$ large enough. In particular, in the pictures in the present paper, we have $A \geq 1$. In the cases considered, $F$ is positive and locally increasing in a right neighborhood of 0 . If $F$ is not globally increasing, it has a local maximum point, followed by the steady state and then by a local minimum point, and after that $F$ grows monotonically to infinity. 


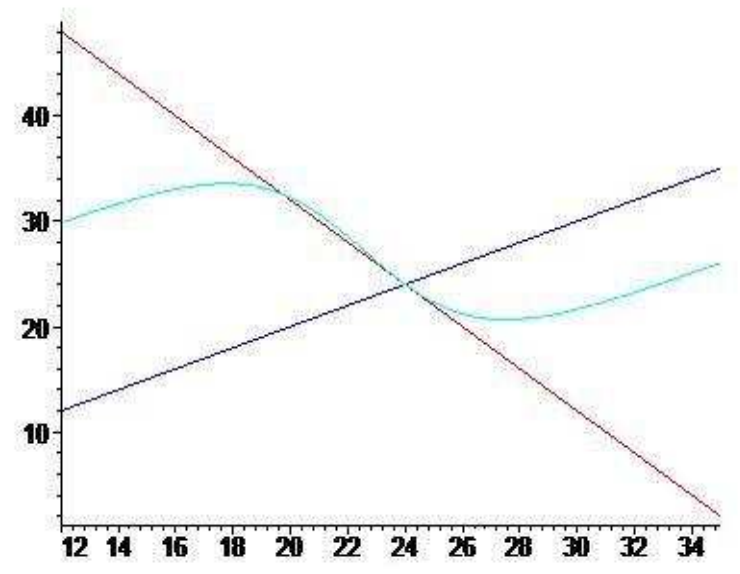

Figure 6: The maps describing the dynamics for the linear and nonlinear cases (in red and cyan, respectively), depicted in a neighborhood of $Y^{*}$ when $a_{1}=3$ and $a_{2}=1.5$, so that $a_{1}+a_{2}=a_{1} a_{2}$.

Proof. Let us assume at first that $F$ is increasing and show that, given a generic starting point $\bar{x}$ in $\mathbb{R}_{+}$, its forward trajectory will tend to $Y^{*}$. Since $F(0)>0$ and $Y^{*}$ is the unique fixed point of $F$, then by continuity, $F(x)>x$, for every $x<Y^{*}$, and $F(x)<x$, for every $x>Y^{*}$. Hence, if $0 \leq \bar{x}<Y^{*}$, then $F^{n}(\bar{x})$ will tend increasingly towards $Y^{*}$ as $n \rightarrow \infty$, while if $\bar{x}>Y^{*}$, then $F^{n}(\bar{x})$ will tend decreasingly towards $Y^{*}$ as $n \rightarrow \infty$.

If the map $F$ is not increasing, let us consider a generic starting point $\bar{x}$ in $\mathbb{R}_{+}$and show that its trajectory will eventually remain in $I$. If $\bar{x} \in I$, then by construction its forward orbit will be trapped inside $I$, as well. Let us now proceed with the two remaining cases, i.e., $\bar{x}<m^{\prime}$ and $\bar{x}>M^{\prime}$. Since $Y^{*} \in I$ and by continuity $F(x)>x$, for every $x<Y^{*}$, and $F(x)<x$, for every $x>Y^{*}$, if $\bar{x}<m^{\prime}$, then its iterates will approach $I$ in a strictly increasing way, while if $\bar{x}>M^{\prime}$, then its iterates will approach $I$ in a strictly decreasing way. Once that a forward iterate of $\bar{x}$ lies in $I$, then by construction all its subsequent iterates will be trapped inside $I$, as well. This concludes the proof.

As we shall see in what follows, in the absorbing interval many different dynamic behaviors may arise. We depicted in Figure 9 the absorbing interval in the case the map $F$ is not increasing, and in fact in that graph it holds $F^{\prime}\left(Y^{*}\right)<-1$. Notice that, in Proposition 3.2, the first scenario (i.e., $F$ strictly increasing) may be seen as a limit case of the second framework, in which the absorbing set collapses into a unique point, that is, the steady state.

We conclude the present section by illustrating in some pictures the possible behaviors of the map $F$. In Figure 10 (A) we show the case in which $F$ is strictly increasing and thus the convergence to $Y^{*}$ is monotone. In Figure 10 (B) we consider 


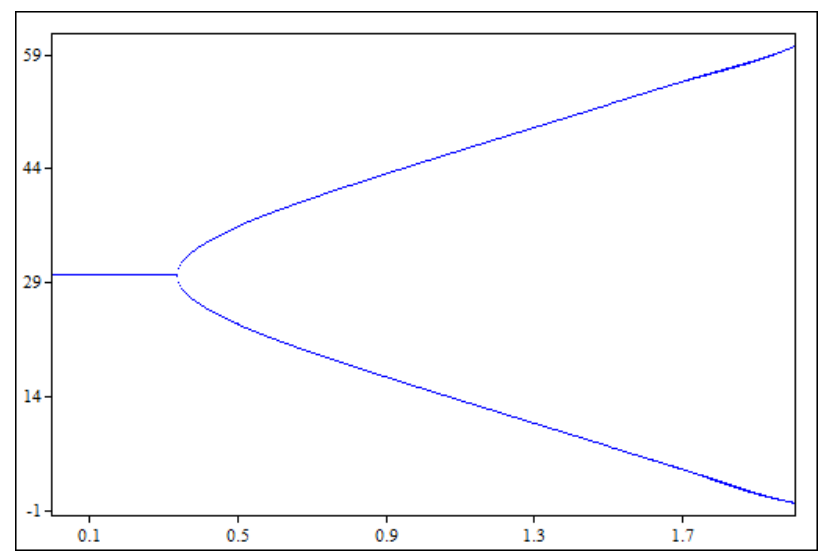

Figure 7: The bifurcation diagram w.r.t. $a_{1}=a_{2} \in[0,2]$ for the map $F$ with $A=12, \gamma=30$ and $c=0.6$.

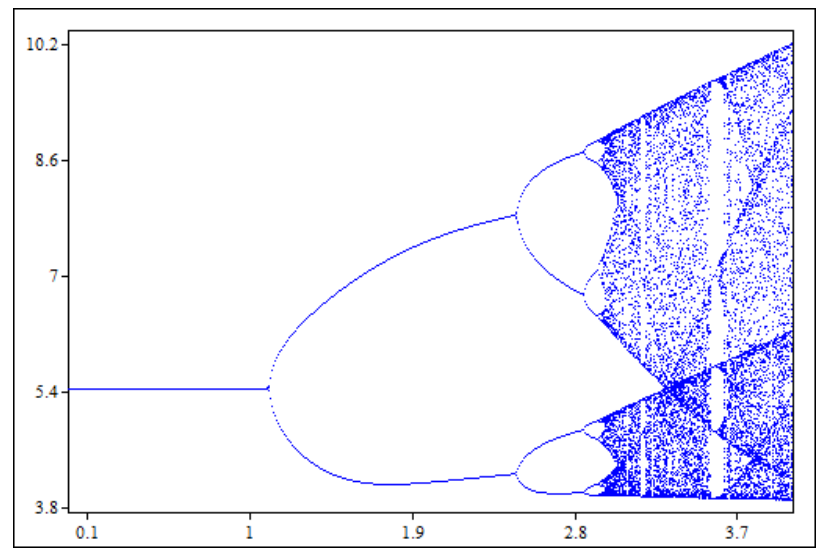

Figure 8: The bifurcation diagram w.r.t. $a_{1} \in[0,4]$ for the map $F$ with $A=5$, $a_{2}=\frac{4}{3}, \gamma=3$ and $c=0.9$.

the framework in which, having increased $\gamma$, the map $F$ is no more globally increasing and the convergence to $Y^{*}$ is oscillatory in nature. Finally, in Figure 10 (C) we deal with the case in which $\gamma$ is still larger and the forward orbit of a generic starting point gets trapped in the absorbing interval.

\section{The flip bifurcation and chaotic dynamics}

In Section 3 we have investigated the existence of an absorbing interval, the stability conditions for the steady state and what happens after its destabilization. Now we will describe how such destabilization originates, i.e., through a period-doubling bifurcation when the reactivity parameter is large enough, and we will show how complex dynamics do emerge when that parameter is even larger. 


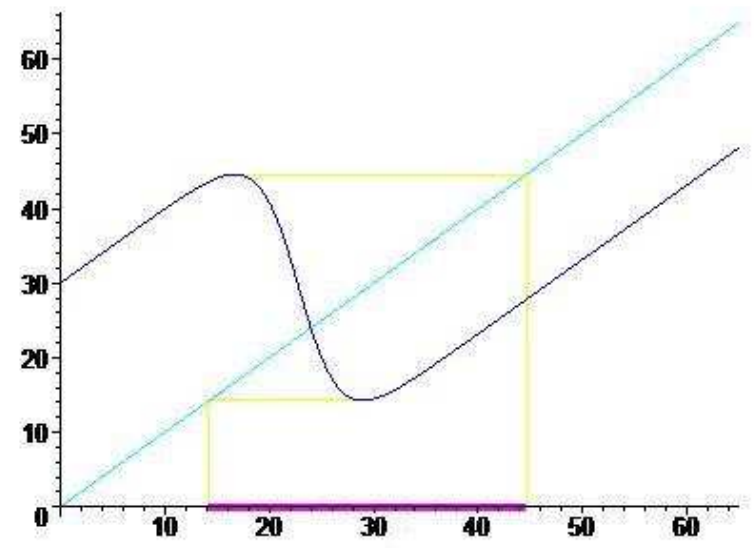

Figure 9: The interval highlighted on the $x$-axis is the absorbing interval $I$ for the map $F$ when $A=12, a_{1}=5, a_{2}=2.8, c=0.6$ and $\gamma=6$. In this case $F^{\prime}\left(Y^{*}\right)<-1$ and the steady state is locally unstable.

We start by verifying that the map $F$ undergoes a flip-bifurcation at the unique steady state $Y^{*}$.

Proposition 4.1 For the map $F$ in (3.1), a flip bifurcation occurs around $Y=Y^{*}$ when $\gamma=\frac{2\left(a_{1}+a_{2}\right)}{a_{1} a_{2}(1-c)}$.

Proof. According to the proof of Proposition 3.1, the steady state $Y=Y^{*}$ is stable when $F^{\prime}\left(Y^{*}\right)>-1$. Then, the map $F$ satisfies the canonical conditions required for a flip bifurcation (see [6]) and the desired conclusion follows. Indeed, when $F^{\prime}\left(Y^{*}\right)=$ -1 , i.e., for $\gamma=\frac{2\left(a_{1}+a_{2}\right)}{a_{1} a_{2}(1-c)}$, then $Y^{*}$ is a non-hyperbolic fixed point; when $\gamma<\frac{2\left(a_{1}+a_{2}\right)}{a_{1} a_{2}(1-c)}$ it is attracting and finally, when $\gamma>\frac{2\left(a_{1}+a_{2}\right)}{a_{1} a_{2}(1-c)}$, it is repelling.

In the proof of Theorem 4.2 we will show the existence of chaos in the sense of Li-Yorke, as described in Conditions (T1) and (T2) in [10, Theorem 1] (from now on, Th1 LY). We recall that result in particular applies to self-maps of an interval $J$, which are continuous and have a periodic point with period 3. Actually, as observed in [10], Th1 LY can be generalized to the case in which $F: J \rightarrow \mathbb{R}$ is a continuous function that does not map the interval $J$ onto itself, as long as it has a period-three point.

Proposition 4.2 Let $F$ be the map in (3.1). Fix $A=12, a_{1}=5, a_{2}=2.8, c=$ $0.6, \gamma=6$ and set $J=[41.946,44.108]$. Then for any point $x \in J$ it holds that $y=F(x), z=F^{2}(x)$ and $w=F^{3}(x)$ satisfy $w \geq x>y>z$ and thus Conditions $(T 1)$ and $(T 2)$ in Th1 LY do hold true. In particular ${ }^{2}$, for any $x \in \operatorname{int}(J)$ it holds

\footnotetext{
${ }^{2}$ Given an interval $I$, we denote its interior by $\operatorname{int}(I)$ and its boundary by $\partial(I)$.
} 


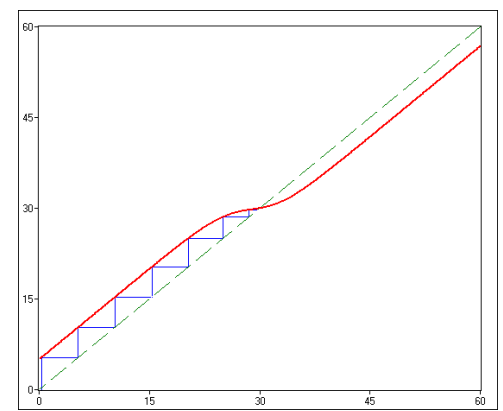

(A)

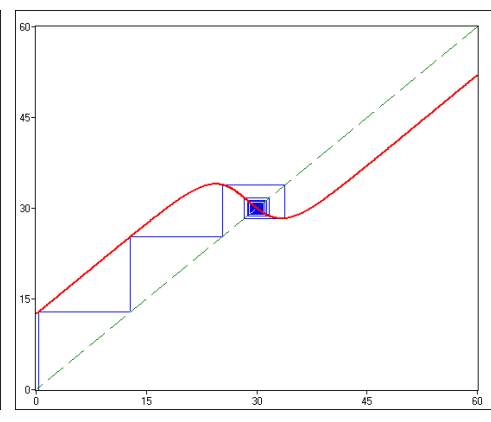

(B)

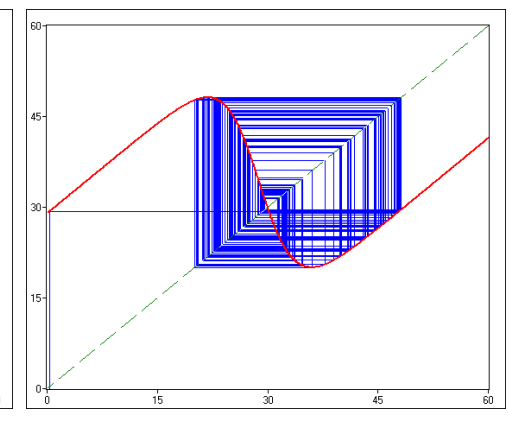

(C)

Figure 10: The first iterate for the map $F$ with $A=12, a_{1}=5, a_{2}=3.2, c=0.6$, and $\gamma=1$ in (A), $\gamma=2.5$ in (B) and $\gamma=5.8$ in (C), respectively.

that $F^{3}(x)>x$, while for any $x \in \partial(J)$ it holds that $F^{3}(x)=x$, that is, the extreme points of $J$ have period three.

Proof of Proposition 4.2: We show that the chain of inequalities $w \geq x>y>z$ is satisfied on $J$ by plotting in Figure 11 the graphs of the identity map in blue, of $F$ in red, of $F^{2}$ in green and of $F^{3}$ in cyan. A direct inspection of the picture shows that it is possible to apply Th1 LY on $J$ and thus we immediately get the desired conclusions.

Notice that in the statement of Proposition 4.2 we have fixed some particular parameter values. However, we stress that the result is robust, as the same conclusions hold for several different sets of parameter values, as well. Moreover, once that a result analogous to Proposition 4.2 is proven for a certain parameter configuration, by continuity, the same conclusions still hold, suitably modifying the interval $J$, also for small variations in those parameters. Hence, Proposition 4.2 actually allows to infer the existence of Li-Yorke chaos for the map $F$ when $\gamma$ lies in a neighborhood of 6 and for some suitable corresponding values of the other parameters.

Notice that both in the statement of Proposition 4.2 and in Figure 11 we considered the same parameter values like in the bifurcation diagram in Figure 4 (except for $\gamma$ there varying on $[1,9]$ ), in order to show that when $\gamma=6$ both the computer simulations and Proposition 4.2 confirm the presence of chaos.

\section{The extended model: endogenous government spending}

In the present section, we present a suitable modification of the model so far analyzed. In fact, instead of an exogenous government expenditure $G=\bar{G}$, in analogy to what 


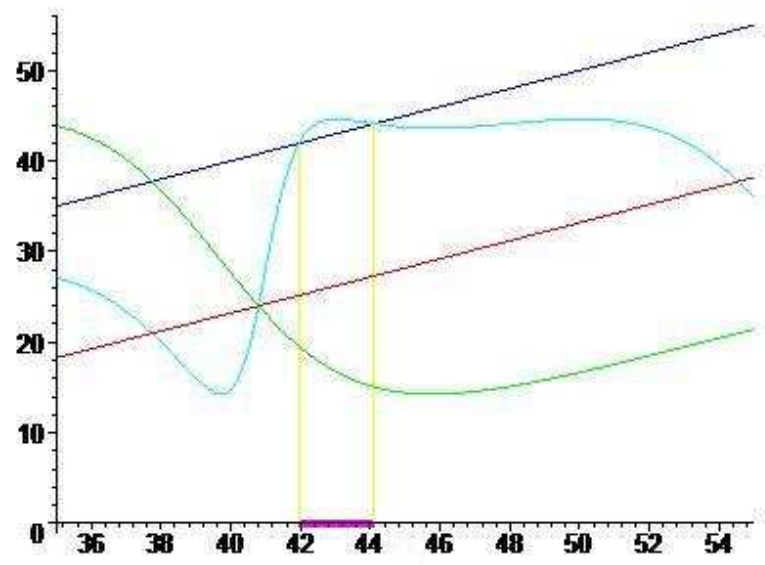

Figure 11: The identity map (in blue) and the first three iterates of the map $\mathrm{F}$ (in red, green and cyan, respectively) for $A=12, a_{1}=5, a_{2}=2.8, c=0.6$ and $\gamma=6$. The interval highlighted on the $x$-axis is $J$ from Proposition 4.2 .

done with consumption, we assume

$$
G_{t}=\bar{G}+\sigma\left(Y^{F}-Y_{t}\right)
$$

where $Y^{F}$ and $\sigma$ are positive constants denoting respectively the income target of the fiscal authority and the reactivity of the fiscal policy with respect to the difference between the income target and the current income. With this new formulation, the expression for the excess demand becomes

$$
E_{t}=Z_{t}-Y_{t}=A+c Y_{t}+\sigma\left(Y^{F}-Y_{t}\right)-Y_{t}
$$

and the corresponding dynamic equation is given by

$$
Y_{t+1}=Y_{t}+\gamma a_{2}\left(\frac{a_{1}+a_{2}}{a_{1} e^{-\left(A+\sigma Y^{F}-Y_{t}(1-c+\sigma)\right)}+a_{2}}-1\right) \text {. }
$$

Similarly to what done in Section 3, we introduce the map $F_{\sigma}: \mathbb{R}_{+} \rightarrow \mathbb{R}$ defined as

$$
F_{\sigma}(Y)=Y+\gamma a_{2}\left(\frac{a_{1}+a_{2}}{a_{1} e^{-\left(A+\sigma Y^{F}-Y(1-c+\sigma)\right)}+a_{2}}-1\right),
$$

associated to the dynamic equation in (5.2).

In equilibrium, the excess demand in (5.1) vanishes and thus we find the expression for the steady state in the next result. 
Proposition 5.1 The dynamical system generated by the map $F_{\sigma}$ in (5.3) has the unique steady state

$$
Y_{\sigma}^{*}=\frac{A+\sigma Y^{F}}{1-c+\sigma}
$$

It holds that

$$
\frac{\partial Y_{\sigma}^{*}}{\partial \sigma}=\frac{(1-c) Y^{F}-A}{(1-c+\sigma)^{2}},
$$

which is positive if and only if $Y^{F}>\frac{A}{1-c}=Y^{*}$ in (2.4), and

$$
\frac{\partial Y_{\sigma}^{*}}{\partial Y^{F}}=\frac{\sigma}{1-c+\sigma}
$$

which is always positive.

We now analyze the stability of the steady state in Proposition 5.2.

Some comments on the sign of the derivatives in Proposition 5.1 can be found in the subsequent discussion.

Proposition 5.2 The steady state $Y_{\sigma}^{*}$ in (5.4) is stable if and only if

$$
\gamma(1-c+\sigma)<\frac{2\left(a_{1}+a_{2}\right)}{a_{1} a_{2}} .
$$

Proof. The result immediately follows by solving the chain of inequalities $-1<$ $F_{\sigma}^{\prime}\left(Y_{\sigma}^{*}\right)<1$ with respect to $\gamma(1-c+\sigma)$. In fact, it is immediate to find that $F_{\sigma}^{\prime}\left(Y_{\sigma}^{*}\right)=$ $1-\frac{\gamma a_{1} a_{2}(1-c+\sigma)}{a_{1}+a_{2}}$ and thus, in order to get the stability of the steady state, we only need to impose $F_{\sigma}^{\prime}\left(Y_{\sigma}^{*}\right)>-1$.

Hence, Proposition 5.2 allows to infer that the steady state is stable when both $\gamma$ and $\sigma$ are small enough. In particular, the stability condition in Proposition 5.2 may be rewritten in terms of $\gamma$ as follows: $\gamma<\frac{2\left(a_{1}+a_{2}\right)}{a_{1} a_{2}(1-c+\sigma)}$. Setting $\overline{\bar{\gamma}}=\frac{2\left(a_{1}+a_{2}\right)}{a_{1} a_{2}(1-c+\sigma)}$ and comparing it with the threshold $\overline{\bar{\gamma}}=\frac{2\left(a_{1}+a_{2}\right)}{a_{1} a_{2}(1-c)}$ encountered in Section 3, we notice that the presence of $\sigma$ makes the stability region smaller with respect to the case of an exogenous government spending.

We illustrate the destabilizing role of $\sigma$ in Figures 12 (A)-(B) below, in which we represent the bifurcation diagrams with respect to $\sigma$ for the first iterate of $F_{\sigma}$ for $A=1$ and $A=4$, respectively, while keeping the other parameters fixed. In both cases, when $\sigma$ increases, the system gets destabilized: notice however that $\frac{\partial Y_{\sigma}^{*}}{\partial \sigma}$ is positive in the first scenario and negative in the second one. In fact, as stated in Proposition 5.1, it holds that $\frac{\partial Y_{\sigma}^{*}}{\partial \sigma}>0$ if and only if $Y^{F}>\frac{A}{1-c}=Y^{*}$, that is, the steady state value increases (decreases) when $\sigma$ increases if and only if the target value $Y^{F}$ is larger than the steady state value in absence of endogenous government spending. 


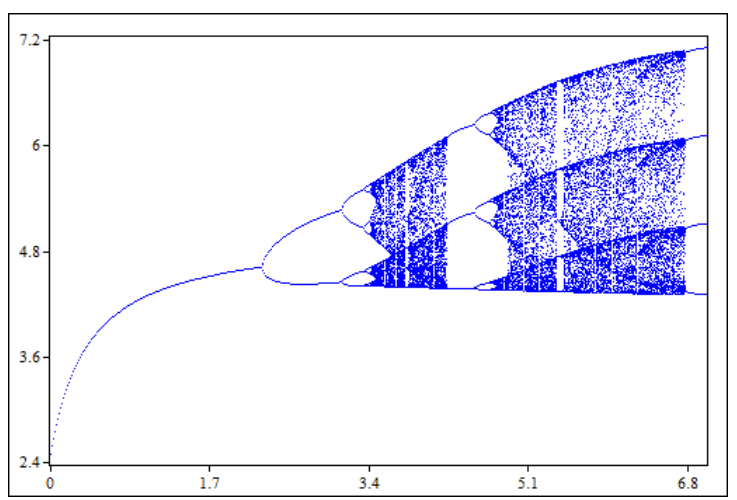

(A)

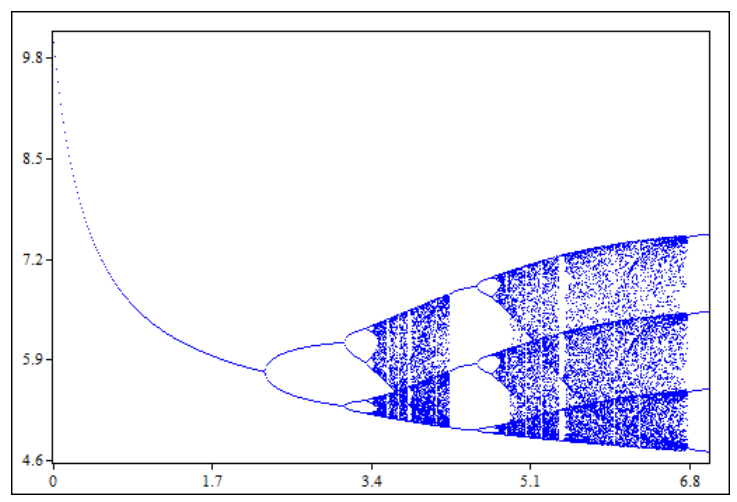

(B)

Figure 12: The bifurcation diagrams w.r.t. $\sigma \in[0,7]$ for the map $F_{\sigma}$ with $a_{1}=3$, $a_{2}=1, c=0.6, \gamma=1, Y^{F}=5$, and $A=1$ in (A) and $A=4$ in (B), respectively.

In regard to the dependence of the steady state on $Y^{F}$, we saw in Proposition 5.1 that instead $\frac{\partial Y_{\sigma}^{*}}{\partial Y^{F}}>0$. Hence we stress that, although by Proposition $5.2 Y^{F}$ does not influence the stability of the steady state, that parameter does influence its position. In particular, increasing $Y^{F}$ moves $Y_{\sigma}^{*}$ to the right. Through some pictures (see Figures 13 (A)-(C) below) we show that $Y^{F}$ also influences the position of the critical points, that simultaneously emerge when the map $F$ is not globally increasing ${ }^{3}$. In fact, increasing $Y^{F}$ moves, not only the steady state, but also the local minimum and maximum points to the right on the $x$-axis, and consequently the absorbing interval gets moved to the right, too, together with the attractor possibly contained in it. This means that, no matter what kind of attractor the system has, the income value levels corresponding to that attractor increase when the income target increases.

In order to illustrate such phenomena, we represent in Figures 13 (A)-(C) below the first iterate of $F_{\sigma}$ for $Y^{F}=1, Y^{F}=30$ and $Y^{F}=60$, respectively, while keeping the other parameters fixed.

Also in this context with an endogenous government spending, we obtain a result

\footnotetext{
${ }^{3}$ In fact, similarly to what argued for the map $F$ in $(3.1)$, it is possible to show that
}

$$
F_{\sigma}(0)=\gamma a_{2}\left(\frac{a_{1}+a_{2}}{a_{1} e^{-\left(A+\sigma Y^{F}\right)}+a_{2}}-1\right)>\gamma a_{2}\left(\frac{a_{1}+a_{2}}{a_{1}+a_{2}}-1\right)=0
$$

and

$$
F_{\sigma}^{\prime}(0)=1-\frac{\gamma a_{1} a_{2}\left(a_{1}+a_{2}\right) e^{-\left(A+\sigma Y^{F}\right)}(1-c+\sigma)}{\left(a_{1} e^{-\left(A+\sigma Y^{F}\right)}+a_{2}\right)^{2}},
$$

which is positive for any $A$ large enough. In the cases considered in the present paper, $F_{\sigma}$ is positive and locally increasing in a right neighborhood of 0 . When $F_{\sigma}$ is not globally increasing, it has a local maximum point, followed by the steady state and then by a local minimum point, and after that $F_{\sigma}$ grows monotonically to infinity. 


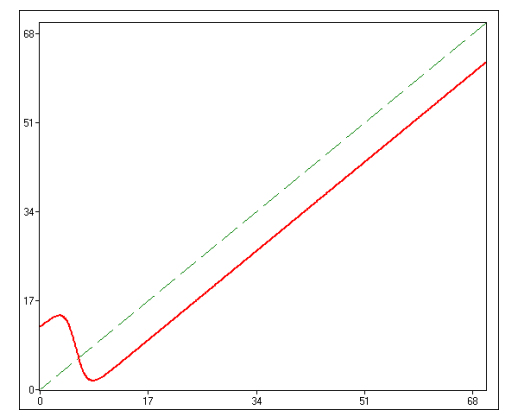

(A)

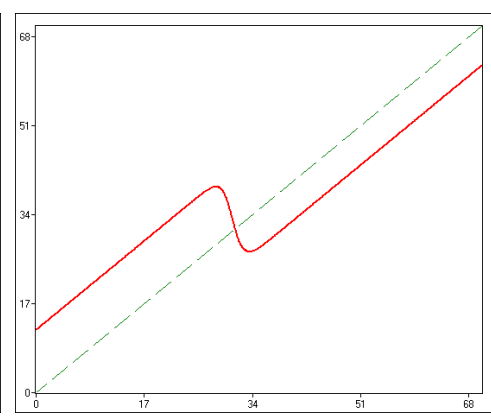

(B)

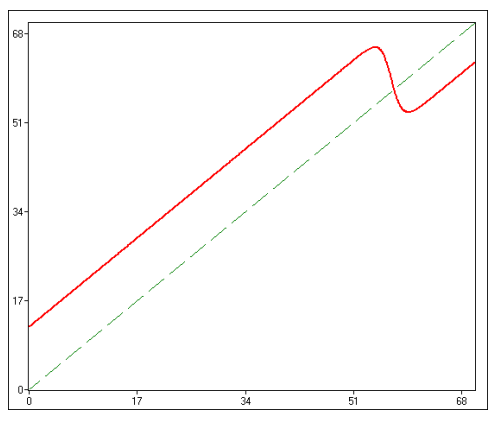

(C)

Figure 13: The graph of the first iterate of the map $F_{\sigma}$ with $A=6, a_{1}=4, a_{2}=$ 2.5, $c=0.85, \gamma=3, \sigma=1$, and $Y^{F}=1$ in (A), $Y^{F}=30$ in (B) and $Y^{F}=60$ in (C), respectively.

similar to Proposition 3.2, whose precise statement reads as follows:

Proposition 5.3 If the map $F_{\sigma}$ in (5.3) is increasing, then the generated dynamical system is globally stable. Else, call $m$ and $M$ the unique positive local minimum point and local maximum point of the map $F_{\sigma}$, respectively, and set $m^{\prime}:=F_{\sigma}(m)$ and $M^{\prime}:=F_{\sigma}(M)$. Then the compact interval $I=\left[m^{\prime}, M^{\prime}\right]$ is "globally absorbing", i.e., for all $\bar{x} \in \mathbb{R}_{+}$there exists $\bar{n} \in \mathbb{N}$ such that $F_{\sigma}^{\bar{n}}(\bar{x}) \in I$ and for any $x \in I, F_{\sigma}^{n}(x) \in I$, for all $n \in \mathbb{N}$.

We omit the proof as it follows the same steps used to prove Proposition 3.2.

The absorbing interval in the case the map $F_{\sigma}$ is not increasing is depicted in Figure 14.

Let us now make precise the statement of a result analogous to Proposition 4.1, according to which the map $F_{\sigma}$ undergoes a flip-bifurcation at the unique steady state $Y_{\sigma}^{*}$. Again, we omit the proof as it is analogous to that of Proposition 4.1.

Proposition 5.4 For the map $F_{\sigma}$ in (5.3), a flip bifurcation occurs around $Y=Y_{\sigma}^{*}$ when $\gamma=\frac{2\left(a_{1}+a_{2}\right)}{a_{1} a_{2}(1-c+\sigma)}$.

We now show the existence of Li-Yorke chaos for the scenario with endogenous government spending.

Proposition 5.5 Let $F_{\sigma}$ be the map in (5.3). Fix $A=1, a_{1}=3, a_{2}=1, c=$ $0.6, \gamma=1, Y^{F}=5, \sigma=4.5$ and set $J=[5.965,6.238]$. Then for any point $x \in J$ it holds that $y=F_{\sigma}(x), z=F_{\sigma}^{2}(x)$ and $w=F_{\sigma}^{3}(x)$ satisfy $w \geq x>y>z$ and thus Conditions (T1) and (T2) in Th1 LY do hold true. In particular, for any $x \in \operatorname{int}(J)$ it holds that $F_{\sigma}^{3}(x)>x$, while for any $x \in \partial(J)$ it holds that $F_{\sigma}^{3}(x)=x$, that is, the extreme points of $J$ have period three. 


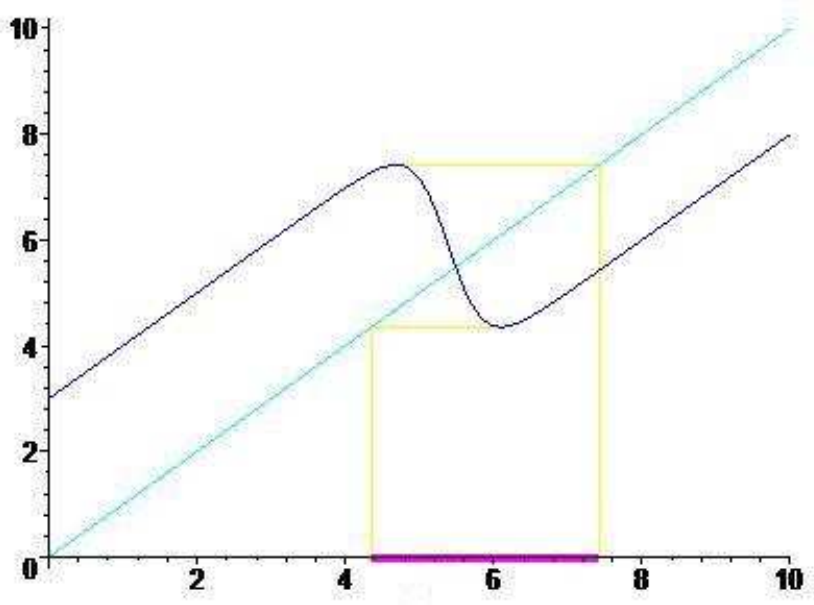

Figure 14: The interval highlighted on the $x$-axis is the absorbing interval $I$ for the map $F_{\sigma}$ when $A=4, a_{1}=3, a_{2}=2, c=0.6, \gamma=1, \sigma=3.7$ and $Y^{F}=5$.

Proof. In analogy with the proof of Proposition 4.2, we show that the chain of inequalities $w \geq x>y>z$ is satisfied on $J$ by plotting in Figure 15 the graphs of the identity map in blue, of $F_{\sigma}$ in red, of $F_{\sigma}^{2}$ in green and of $F_{\sigma}^{3}$ in cyan. A direct inspection of the picture shows that it is possible to apply Th1 LY on $J$ and thus we immediately get the desired conclusions.

Similarly to what observed in relation to Proposition 4.2, by continuity, Proposition 5.5 actually allows to infer the existence of Li-Yorke chaos for the map $F_{\sigma}$ when $\sigma$ lies in a neighborhood of 4.5 and for some suitable corresponding values of the other parameters.

Notice that both in the statement of Proposition 5.5 and in Figure 15 we considered the same parameter values like in the bifurcation diagram in Figure 12 (A) (except for $\sigma$ there varying on $[0,7]$ ), in order to show that when $\sigma=4.5$ both the computer simulations and Proposition 5.5 confirm the presence of chaos. In this regard, we stress that, for instance, we could have written Proposition 5.5 with $A=4, a_{1}=3, a_{2}=1, c=0.6, \gamma=1, Y^{F}=5, \sigma=6$ and $J=[6.376,6.951]$ as well, in agreement with the chaotic behavior detected in Figure 12 (B) for those parameter values.

\section{Global analysis}

In Sections 3 to 5 , we presented some theoretical results about the dynamics of our systems, both with exogenous and endogenous government spending. We saw that in both frameworks different kinds of dynamics can occur, such as the existence of 


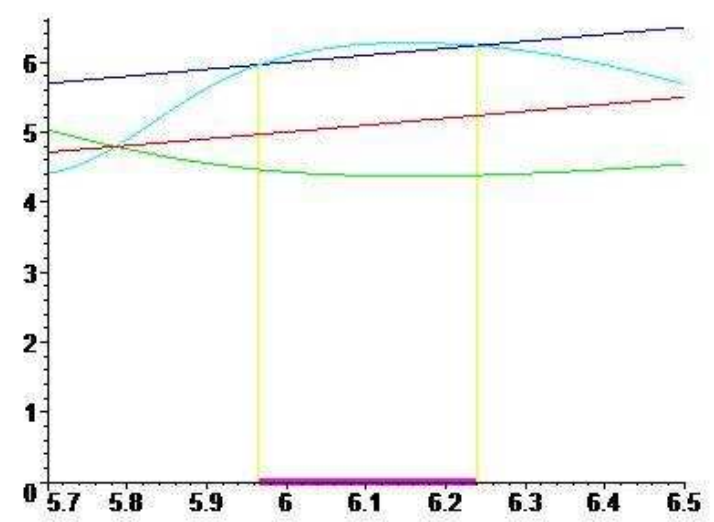

Figure 15: The identity map (in blue) and the first three iterates of the map $F_{\sigma}$ (in red, green and cyan, respectively) for $A=1, a_{1}=3, a_{2}=1, c=0.6, \gamma=1, Y^{F}=5$ and $\sigma=4.5$. The interval highlighted on the $x$-axis is $J$ from Proposition 5.5.

absorbing intervals, stable steady states, periodic cycles and chaotic behaviors. In this section we investigate, using bifurcation and time series diagrams, the global behavior of the system as the parameter $\sigma$ increases.

In particular, in Figure 16 we illustrate a numerical example in which, from a stable steady state to a chaotic attractor, a multistability scenario occurs. Indeed we show that there exists a sufficiently large value of $\sigma$ for which we have the coexistence of a stable three-orbit with a chaotic attractor in four pieces. In Figure 17 we present a magnification of Figure 16, in which we better illustrate that coexistence phenomenon. Moreover, in Figures 18 (A)-(B) we show the time series corresponding to the periodthree cycle with initial condition $Y(0)=1$ and to the chaotic attractor in four pieces with initial condition $Y(0)=3$, respectively.

\section{Conclusions}

In this paper we showed how a rich variety of dynamical behaviors can emerge in the standard Keynesian income-expenditure model when a nonlinearity is introduced, both in the cases with and without endogenous government spending. A specific sigmoidal functional form has been used for the adjustment mechanism of income with respect to the excess demand, in order to bound the income variation. With the aid of analytical and numerical tools, we investigated the stability conditions, bifurcations, as well as periodic and chaotic dynamics. Globally, we studied multistability phenomena, i.e., the coexistence of different kinds of attractors.

Here we proposed a simple model, in order to improve our knowledge about the role of nonlinearities in the emergence of complex behaviors. Of course, it may be extended 


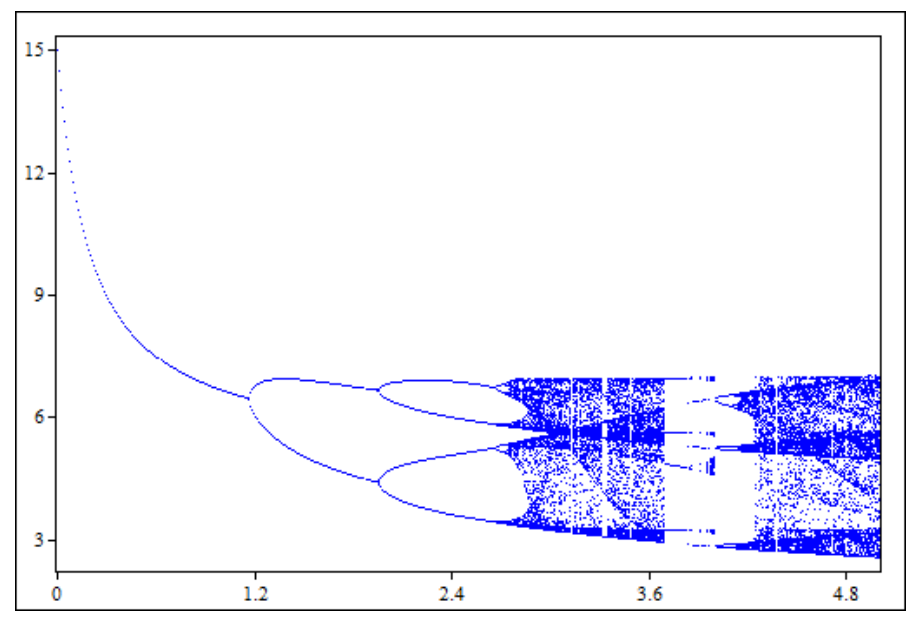

Figure 16: The bifurcation diagram w.r.t. $\sigma \in[0,5]$ for the map $F_{\sigma}$ with $A=3, a_{1}=$ $3, a_{2}=4.6935, c=0.8, \gamma=0.8$ and $Y^{F}=5$, which highlights a multistability phenomenon characterized by the coexistence of a stable three-orbit with a chaotic attractor in four pieces.

in various directions, for instance introducing further nonlinearities in the definition of the relevant economic variables. Moreover, subsequent work should focus on extensions of the present model in view of considering money supply, interest rate and the general price level, too, aspects that are taken into account in more sophisticated classes of models, such as the IS-LM and AD-AS frameworks. 


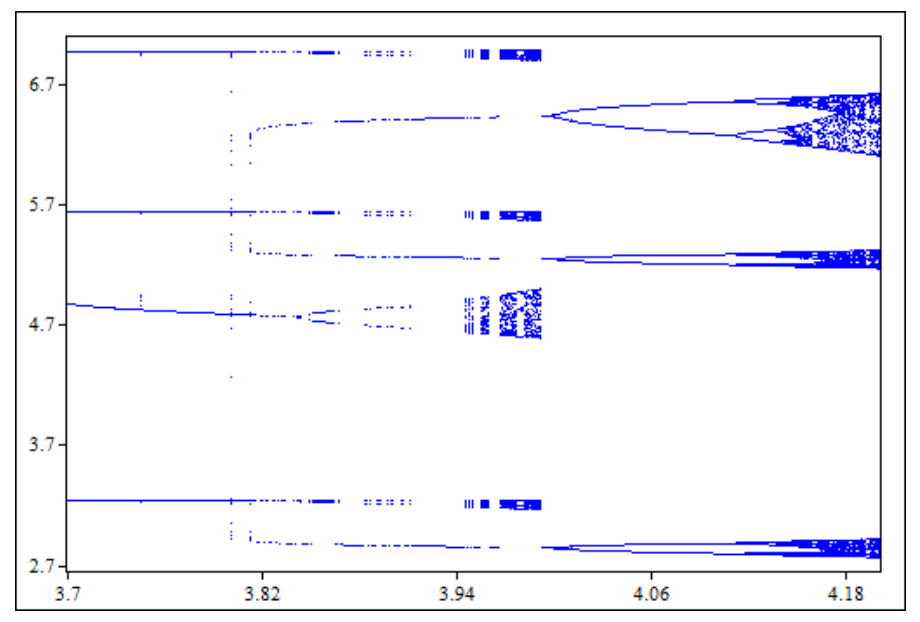

Figure 17: A magnification of Figure 16 for $\sigma \in[3.7,4.2]$, in order to better show the coexistence of a stable three-orbit with a chaotic attractor in four pieces.

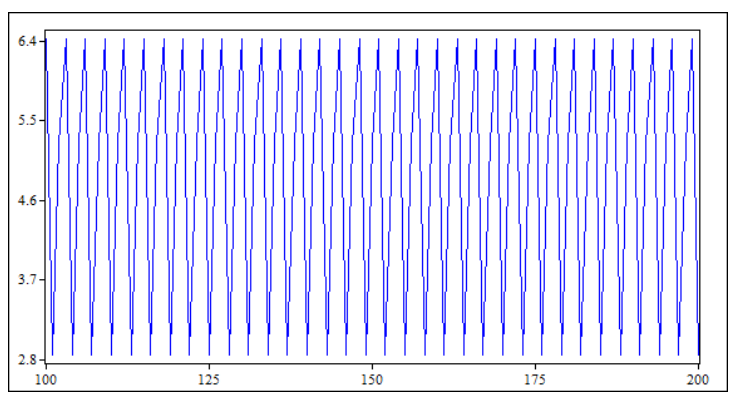

(A)

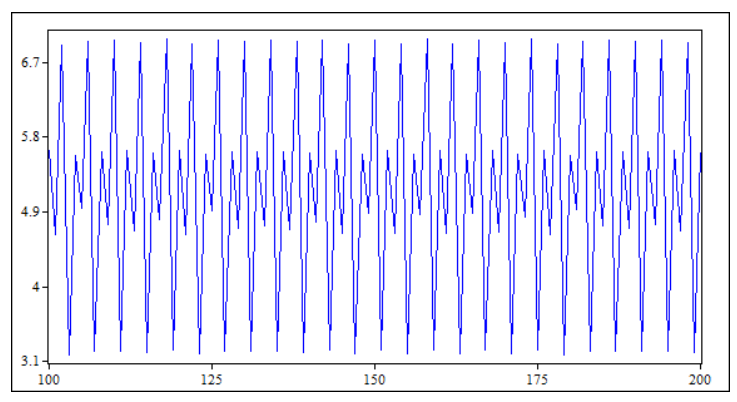

(B)

Figure 18: The time series corresponding to the period-three cycle in Figures 16 and 17 in $(\mathrm{A})$ and the chaotic attractor in four pieces in $(\mathrm{B})$, with initial conditions $Y(0)=1$ and $Y(0)=3$, respectively.

\section{References}

[1] L. De Cesare, M. Sportelli, A dynamic IS-LM model with delayed taxation revenues, Chaos, Solitons and Fractals 25 (2005) 233-244.

[2] L. De Cesare, M. Sportelli, Fiscal policy lags and income adjustment processes, Chaos, Solitons and Fractals 45 (2012) 433-438.

[3] L. Fanti, P. Manfredi, Chaotic business cycles and fiscal policy: An IS-LM model with distributed tax collection lags, Chaos, Solitons and Fractals 32 (2007) 736744 .

[4] B.S. Ferguson, G.C. Lim, Dynamic Economic Models in Discrete Time: Theory and Empirical Applications, Routledge, London, 2003. 
[5] J.-M. Grandmont, Nonlinear Economic Dynamics. Academic Press, New York, 1988.

[6] J.K. Hale, H. Koçak, Dynamics and Bifurcations, Texts in Applied Mathematics, vol. 3, Springer-Verlag, New York, 1991.

[7] C. Hommes, Behavioral Rationality and Heterogeneous Expectations in Complex Economic Systems, Cambridge University Press, Cambridge, 2013.

[8] F.E. Kydland, E.C. Prescott, Time to build and aggregate fluctuations, Econometrica 50 (1982) 1345-1370.

[9] F.E. Kydland, E.C. Prescott, The econometrics of the general equilibrium approach to business cycles, The Scandinavian Journal of Economics 93 (1991) $161-178$.

[10] T.-Y. Li, J.A. Yorke, Period three implies chaos, Amer. Math. Monthly 82 (1975) 985-992.

[11] A. Medio, Chaotic Dynamics. Theory and Applications to Economics, Cambridge University Press, Cambridge, 1992.

[12] M. Wegener, F. Westerhoff, Evolutionary competition between prediction rules and the emergence of business cycles within Metzler's inventory model, Journal of Evolutionary Economics 22 (2012) 251-273.

[13] F. Westerhoff, Interactions between the real economy and the stock market: a simple agent-based approach, Discrete Dynamics in Nature and Society (2012), Article ID 504840. 